\title{
Evaluation of the solubility of 11-keto- $\beta$-boswellic acid and its histological effect on the diabetic mice liver using a novel technique
}

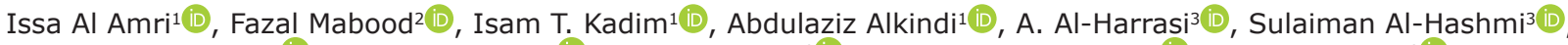 \\ Ghulam Abbas ${ }^{1}$, Ahmed Hamaed ${ }^{1}$ (D), Basant Ahmed ${ }^{3}$ (D), Jawaher Al-Shuhaimi ${ }^{1}$, Samera Khalaf ${ }^{3}$ (D) and \\ Jamaluddin Shaikh ${ }^{4}$ (D)
}

1. Department of Biological Sciences and Chemistry, College of Arts and Sciences, University of Nizwa, PO Box 33, PC 616, Birkat Al-Mouz, Nizwa, Sultanate of Oman; 2. Institute of Chemical Sciences, University of Swat KP, Pakistan; 3. Natural and Medical Sciences Research Center, University of Nizwa, PO Box 33, PC 616, Birkat Al-Mouz, Nizwa, Sultanate of Oman; 4. School of Pharmacy, College of Pharmacy and Nursing, University of Nizwa, PO Box 33, PC 616, Birkat Al-Mouz, Nizwa, Sultanate of Oman.

Corresponding author: Isam T. Kadim, e-mail: isam@unizwa.edu.om

Co-authors: IA: issa.alamri@unizwa.edu.om, FM: mehboob@unizwa.edu.om, AbA: alkindi@unizwa.edu.om, AA: aharrasi@unizwa.edu.om, SA: sahashmi@unizwa.edu.om, GA: abbashej@unizwa.edu.om, AH: ahmed.hamaed@unizwa.edu.om,BA: Bosy.bt@gmail.com, JA: 09736847@uofn.edu.om, SK: khalafsamera@gmail.com, JS: jamaluddin@unizwa.edu.om

Received: 31-01-2021, Accepted: 17-05-2021, Published online: 12-07-2021

doi: www.doi.org/10.14202/vetworld.2021.1797-1803 How to cite this article: Al Amri I, Mabood F, Kadim IT, Alkindi A, Al-Harrasi A, Al-Hashmi S, Abbas G, Hamaed A, Ahmed B, Al-Shuhaimi J, Khalaf S, Shaikh J (2021) Evaluation of the solubility of 11 -keto- $\beta$-boswellic acid and its histological effect on the diabetic mice liver using a novel technique, Veterinary World, 14(7): 1797-1803.

\begin{abstract}
Background and Aim: The literature is scant on the effect of 11-keto- $\beta$-boswellic acid (KBA) on the liver of diabetes-induced mice. This study was designed to develop a rapid, sensitive, accurate, and inexpensive detection technique for evaluating the solubility of KBA obtained from the gum resin of Omani frankincense (Boswellia sacra) in the liver of streptozotocininduced diabetic mice using Fourier transform infrared (FTIR) reflectance spectroscopy coupled with principal components analysis (PCA). It also aimed to investigate the effect of KBA on histological changes in the hepatocytes of diabetic mice.

Materials and Methods: Eighteen mice were assigned to the healthy control group, the diabetic control group, or the KBAtreated diabetic group. Liver tissue samples from all groups were scanned using an FTIR reflectance spectrophotometer in reflection mode. FTIR reflectance spectra were collected in the wavenumber range of $400-4000 \mathrm{~cm}^{-1}$ using an attenuated total reflectance apparatus.

Results: FTIR reflectance spectra were analyzed using PCA. The PCA score plot, which is an exploratory multivariate data set, revealed complete segregation among the three groups' liver samples based on changes in the variation of wavenumber position in the FTIR reflectance spectra, which indicated a clear effect of KBA solubility on treatments. Histological analysis showed an improvement in the liver tissues, with normal structures of hepatocytes exhibiting mild vacuolation in their cytoplasm.

Conclusion: KBA improved the morphology of liver tissues in the diabetic mice and led to complete recovery of the damage observed in the diabetic control group. FTIR reflectance spectroscopy coupled with PCA could be deployed as a rapid, low-cost, and non-destructive detection method for evaluating treatment effects in diseased liver tissue based on the solubility of KBA.
\end{abstract}

Keywords: 11 -keto- $\beta$-boswellic acid, Fourier transform infrared reflectance, histology, liver, mice, principle component analysis.

\section{Introduction}

Diabetes mellitus (DM) is a chronic metabolic disease characterized by hyperglycemia resulting from insufficient insulin action [1]. In 2020, the International Diabetes Federation (www.idf.org) reported that there were 463 million cases of diabetes worldwide, including 55 million in the Middle East and North Africa region and 291,000 in Oman.

Copyright: Al Amri, et al. Open Access. This article is distributed under the terms of the Creative Commons Attribution 4.0 International License (http://creativecommons.org/licenses/ by/4.0/), which permits unrestricted use, distribution, and reproduction in any medium, provided you give appropriate credit to the original author(s) and the source, provide a link to the Creative Commons license, and indicate if changes were made. The Creative Commons Public Domain Dedication waiver (http:// creativecommons.org/publicdomain/zero/1.0/) applies to the data made available in this article, unless otherwise stated.
In $2017,94,921$ cases of diabetes were reported in Oman (according to the Ministry of Health 2017 Annual report, www.omanobserver.om). Moreover, the number of diabetes cases in the Middle East and North Africa region has been statistically estimated to increase to 108 million by 2045 [2].

DM causes several diseases, such as neurological disorders, coronary artery disease, renal failure, and cerebrovascular disease; limb amputation; blindness; as well as death $[3,4]$. Types 1 and 2 DM, gestational diabetes, impaired glucose tolerance, and impaired fasting glycemia are considered to be the main types of diabetes [1]. Type $1 \mathrm{DM}$ is an autoimmune disorder, whereas type $2 \mathrm{DM}$ is a metabolic disorder [5]. The human liver is the largest internal organ and has multiple functions, including regulation of the 
glucose concentration in physiological and pathological conditions as well as prevention of its excessive fluctuation [6]. Hyperglycemia affects the metabolism of protein, carbohydrates, and lipids, leading to nonalcoholic fatty liver disease (NAFLD) [7]. NAFLD can further progress to nonalcoholic steatohepatitis, cirrhosis, and, ultimately, hepatocellular carcinomas. Research has shown that NAFLD affects up to $70 \%$ to $80 \%$ of people with type $2 \mathrm{DM}$ and up to $30 \%$ to $40 \%$ of people with type $1 \mathrm{DM}[8]$.

Medicinal components extracted from the frankincense plant (Boswellia spp.) have been documented to possess health benefits and pharmaceutical properties, such as antimicrobial, anti-inflammatory, anticancer, antidiabetic, antioxidant, and other analgesic activities [9-14]. The active derivatives of the plant are the boswellic acids (BAs) found in the gum resin of Boswellia spp., which have pharmacologically active pentacyclic triterpene molecules, including $11-k e t o-\beta-B A(K B A)[10,13]$. These active derivatives have been used to treat a number of inflammatory diseases, such as osteoarthritis, chronic colitis, bronchial asthma, pancreatic cells and tumors, human breast cancer cells [15], and hepatocellular carcinomas [16]. Frankincense has been reported to significantly increase the wound contraction rate after the administration of KBA for 16 days in diabetic mice [17]. In another study, diabetic mice injected with KBA for 7 weeks triggered the infiltration of a large number of lymphocytes into the pancreatic islets, and semi-isolated apoptotic cells were observed [18]. Research has also shown a decrease in the blood sugar level of diabetic mice after injection with KBA [19]. Pharmacokinetic studies, however, have evidenced low absorption of KBA in humans and rodents due to its poor water solubility and a strong tendency to self-aggregate [20-23]. One of such studies showed that the concentration of KBA declined with elimination of half-life after $6 \mathrm{~h}$ of oral administration and suggested that medication is required after every $6 \mathrm{~h}$ [23]. Therefore, strategies to improve the bioavailability of KBA need to be established to ensure its effective anti-inflammatory activity.

Fourier transform infrared (FTIR) reflectance spectroscopy is an effective tool used to determine the chemical characteristics of various sample forms, including solids, liquids, and gases. FTIR reflectance presents unique information by addressing cofactor, amino acid, and water molecule properties with high structural parameters and interactions [24]. Its advantages include rapid sample preparation and processing in solid forms for scanning with an FTIR reflectance spectrophotometer and subsequent analysis using a multivariate principal components analysis (PCA) method, which would allow for the development of a PCA model for exploring similarities and differences among samples based on the solubility of the drug compound.

To the best of our knowledge, the effect of intraperitoneal (IP) treatment with KBA on the liver of diabetes-induced mice has not been previously reported. This study analyzed the solubility of KBA extracted from the gum resin of Omani frankincense (Boswellia sacra) in the liver of streptozotocin (STZ)induced diabetic mice and evaluated the effect of KBA on histological changes in the liver tissue under disease conditions using the IP route of administration.

\section{Materials and Methods}

\section{Ethical approval}

The use of the animals was approved by the Animal Ethics Committee at University of Nizwa.

\section{Study period and location}

The study was conducted from September 2017 to July 2019, in DARIS Research Center, University of Nizwa, Oman.

\section{Experimental design and tissue sample preparation}

Eighteen female CD-1 mice (weight, 25-30 g; age, 10-12 weeks) were used in this study. They were initially divided into two groups: The healthy control group (Group 1; $\mathrm{n}=6$ ) and the STZ-induced group (Group 2; $\mathrm{n}=12$ ). Six mice were kept in cages and exposed to a $12 \mathrm{~h} \mathrm{light/dark} \mathrm{cycle,} \mathrm{with} \mathrm{controlled}$ room temperature $\left(24-25^{\circ} \mathrm{C}\right)$ and humidity $(30-35 \%$.). All mice had access to food and water ad libitum. One week after adaptation, Group 1 mice were injected with citrate buffer, whereas Group 2 mice were given an IP injection of STZ (180 mg/ $\mathrm{kg}$ body weight) in $10 \mathrm{mM}$ citrate buffer ( $\mathrm{pH} 4.5)$. Two weeks after the injection with STZ, blood samples were collected from the tail vein of the mice. STZ-induced mice with blood glucose levels $>300 \mathrm{mg} / \mathrm{dL}$ were classified as diabetic mice and further divided into two experimental groups, each consisting of six mice: The diabetic control group and the KBA-treated diabetic group. The diabetic control mice (Group 2) received water only, whereas the diabetic experimental mice received IP injections of KBA dissolved in water at a dose of $25 \mathrm{mg} / \mathrm{kg} / \mathrm{d}$. Four weeks later, after the final treatment, the mice were anesthetized and killed. For light microscopy screening, liver tissues were dissected and immersed in 10\% buffered formaldehyde solution; for FTIR reflectance analysis, liver tissues were frozen at $-80^{\circ} \mathrm{C}$ in liquid nitrogen.

\section{FTIR reflectance spectroscopy}

Each dried liver tissue sample was scanned using an FTIR reflectance spectrophotometer to produce reflectance spectra at five positions, with 16 scans per position to produce 80 spectra per sample. The averages of the 80 spectra per sample were used to build a multivariate PCA model.

\section{PCA}

PCA is an unsupervised data exploration multivariate chemometric method used to show hidden similarities and differences among samples. In this study, Unscrambler X10.3 (CAMO Software, Oslo, Norway) and Microsoft Excel 2016 were used to create a PCA model of the FTIR reflectance spectral 
data. PCA was performed to determine the classification and segregation of the three groups' dried liver tissue samples based on the solubility of KBA. The PCA model was built using the singular value decomposition algorithm. The internal validation of the PCA model was carried out using a leave-one-out, full cross-validation procedure, which involved investigating the maximum of seven principal components.

\section{Preprocessing of near-IR reflectance spectral data}

Before the PCA modeling, unit vector normalization, standard normal variate transformation, and multiplicative scatter correction were employed to correct the multiplicative and additive effects of the spectra. Smoothing is usually applied to eliminate instrumental clutter or background information, and detrending methods are usually implemented to reduce the effects of accumulating data sets from a trend. The first and second derivatives of the spectra (D1 and D2) based on the Savitzky-Golay algorithm, with five smoothing points and polynomial order of 2 , were also implemented to increase the spectral resolution. Derivatives are commonly used to reduce insignificant baseline signals from samples [25].

\section{Light microscopy}

Tissue samples were fixed in $10 \%$ buffered formaldehyde solution for $8 \mathrm{~h}$ and then sliced into small pieces. The tissue slices were dehydrated in graded concentrations of ethyl alcohol, diaphonized in xylol, and then impregnated and embedded in paraffin wax. Sections (thickness, $4 \mu \mathrm{m}$ ) were produced by a rotary microtome and then stained with hematoxylin and eosin. For morphological examination, slides of stained sections were screened using a digital light microscope under $40 \times-1000 \times$.

\section{Results}

\section{FTIR reflectance analysis}

The FTIR reflectance spectra representing solid liver tissue samples from the three study groups were in reflection mode in the wavenumber range of 400$4000 \mathrm{~cm}^{-1}$ (Figure-1). They demonstrated a prominent increase in the peak intensities in the wavenumber range of $950-1650 \mathrm{~cm}^{-1}$ and in that of 2009-3568 $\mathrm{cm}^{-1}$ as KBA doses increased from Groups 1 to 3, which indicated a clear effect of KBA solubility on treatments. The FTIR reflectance spectra of pure KBA demonstrated characteristic peaks at $3437 \mathrm{~cm}^{-1}$ (O-H stretching), $2932 \mathrm{~cm}^{-1}$ (C-H stretching), 1697 $\mathrm{cm}^{-1}$ (C-O stretching of aryl acid), $1453 \mathrm{~cm}^{-1}$ (C-H bending), $1375 \mathrm{~cm}^{-1}$ (COO symmetric stretching of carboxylates), $1240 \mathrm{~cm}^{-1}$ (C-CO-C stretching of aryl ketone), as well as 1025 and $988 \mathrm{~cm}^{-1}$ (ring structures of cyclohexane) [26].

\section{PCA}

PCA is an unsupervised multivariate exploratory data analysis tool that extracts such hidden information as similarities and differences based on variations in the data. Therefore, a PCA model (Figure-2) was built to extract masked information on the FTIR reflectance spectra for classification and discrimination among the three groups' liver tissue samples based on KBA solubility. The PCA score plot (Figure-2) demonstrated a clear classification among the three groups based on the variation in FTIR reflectance peak intensities as well as peak position. The three groups' tissue samples were mapped based on the solubility of KBA. Samples from Groups 1 and 3 were differentiated on the PC- 1 axis, which utilized $58 \%$ of the spectral variation, whereas those from Groups 2 and 3 were differentiated on the PC-2 axis, which had $21 \%$ of the spectral information.

PCA loadings were plotted to determine the part of the spectral variation that contributed to the PCA model (Figure-3). The loading plots for PC-1 demonstrated that the spectral regions in the wavenumber ranges of $950-1650 \mathrm{~cm}^{-1}$ and $2009-3568 \mathrm{~cm}^{-1}$ were the most important regions of the FTIR reflectance spectra and contributed mainly to the PCA model in increasing relevance from Groups 1 to 3, indicating a clear effect of KBA solubility on treatments - as KBA concentration peaks were distinctly elevated in Group 3.

\section{Histopathological analysis}

Liver sections of the healthy mice treated with citrate buffer (Group 1) showed a liver architecture made of classical and portal lobules (Figure-4a). The classical lobules consisted of plates of hepatocytes radiating from a central vein and extending toward portal areas (Figure-4b). The portal lobules (triads) consisted of the hepatic artery, hepatic portal vein, bile duct, and lymphatic vessels (Figure-4). Between the hepatocyte plates were liver sinusoids, which are capillaries that carry blood from the hepatic portal vein, enter the hepatic artery through the portal triads, and then drain into the central vein (Figure- $4 b$ and c).

The histological results for the diabetic mice (Group 2) showed that the classical and portal lobules of liver tissues (Figure-5a) with histopathological changes, including hepatocellular damage in the form of increased vacuolation in the cytoplasm of hepatocytes, appeared as indistinct clear vacuoles, which indicated glycogen infiltration and accumulation (Figure-5b and c). The liver samples from the diabetic mice given IP injections of KBA (Group 3) showed a normal liver architecture (Figure-6a) with mild vacuolation in the cytoplasm of hepatocytes (Figure-6b and c).

\section{Discussion}

DM is a metabolic disorder caused mainly by hyperglycemia and hyperlipidemia; it affects most of the body's vital organs and involves multiple therapies for treatment [27]. Efficient treatments for certain types of diabetes (e.g., type 1 and late-onset autoimmune diabetes) are currently not available, but recent research has shown that the antidiabetic properties 


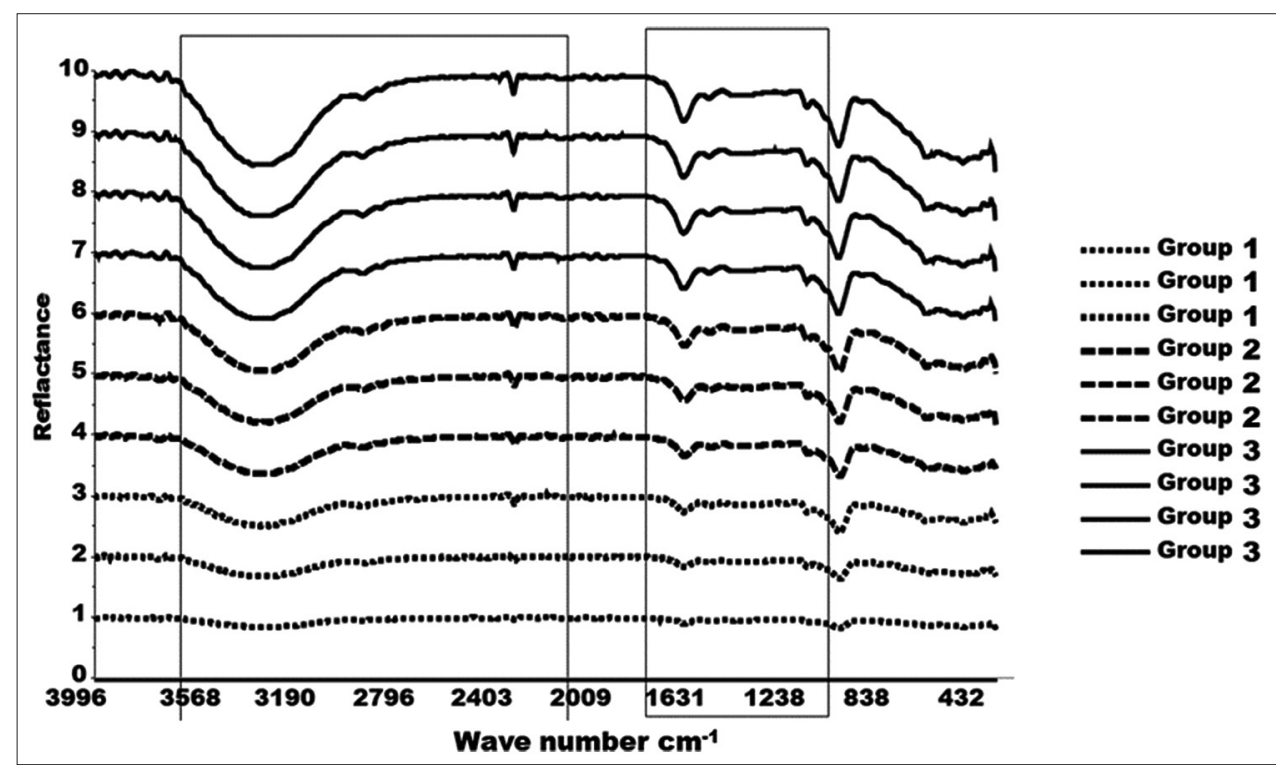

Figure-1: Fourier transform infrared spectra of solid liver tissue samples in reflectance mode for the three groups of mice. Group-1 normal liver tissue, Group-2 diabetic control liver tissue, and Group-3 treated intraperitoneal with 11-keto- $\beta$ boswellic acid tissue.

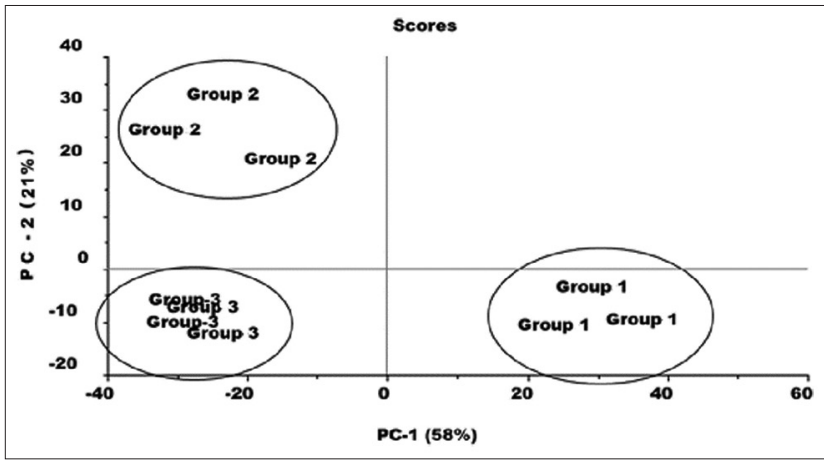

Figure-2: Principle component analysis score plot for three groups of mice liver samples (Group-1 normal liver, Group-2 diabetic control liver, and Group-3 diabetic liver treated intraperitoneal with 11-keto- $\beta$-boswellic acid).

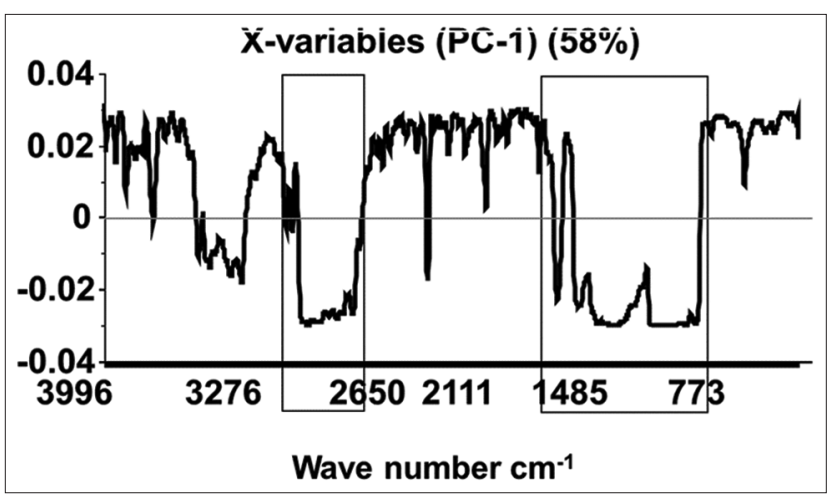

Figure-3: Principle component analysis loading plot.

of BAs, the pharmacologically active constituents of frankincense, render them a viable therapeutic option [12]. The anti-inflammatory properties of BAs have been reported as targets for immune system factors involved in types 1 and $2 \mathrm{DM}$ [28]. However, BA compounds, including $\alpha-\mathrm{BA}$ and $\beta$-BA as well as acetylated $\alpha$-BA and $\beta$-BA, were found to have no effect in inhibiting STZ-mediated hyperglycemia,

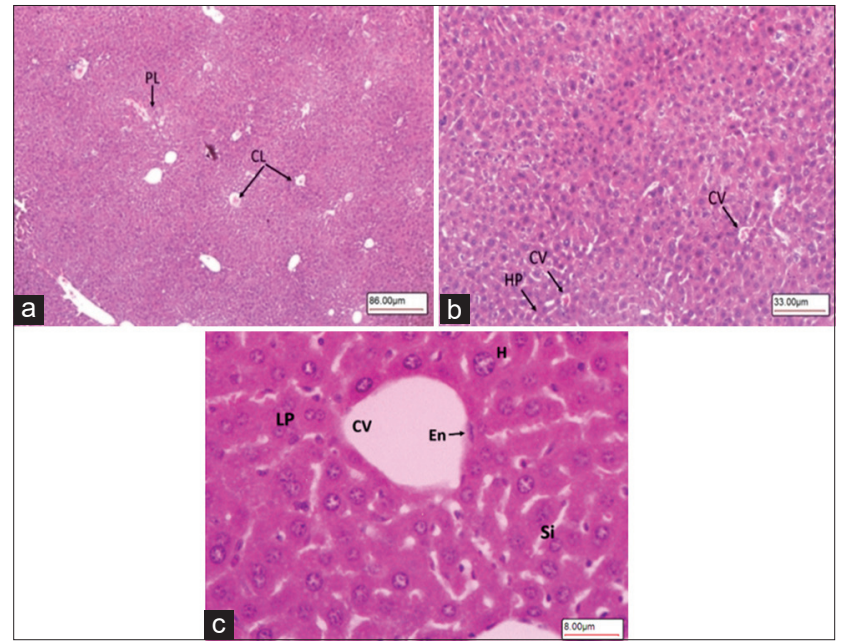

Figure-4: Light microscopy images of hematoxylin-eosin stained liver section showing normal control mice Group-1. (a) Liver histology made up of $\mathrm{CL}$ and PL. (b) $\mathrm{CL}$ consist of plates of HP radiating from a CV. (c) CV lined with En, $\mathrm{H}, \mathrm{LP}$, and liver Si $(40 \times, 100 \times$, and $400 \times$, respectively). $\mathrm{CL}=$ Classical lobules, $\mathrm{PL}=$ Portal lobules, $\mathrm{HP}=$ Hepatocytes, $\mathrm{CV}=$ Central vein, $\mathrm{En}=$ Endothelium, $\mathrm{H}=$ Hepatocytes, $\mathrm{LP}=$ Liver hepatocyte plates, $\mathrm{Si}=$ Sinusoids.

whereas KBA and 3-O-acetyl-KBA were identified as key players in the prevention of diabetes. These isolated compounds, KBA and 3-O-acetyl-KBA, prevented the occurrence of autoimmune reactions as well as insulitis and reduced hyperglycemia in STZ-induced diabetes models [19]. Pharmacokinetic studies of Boswellia spp. extracts reported the poor bioavailability of these compounds in both humans and rats due to their poor water solubility and high lipophilicity $[29,30]$. Research has also shown that permeability-associated barriers that compromise the oral bioavailability of KBA include its gastrointestinal volatility, mediated intestinal metabolism, 


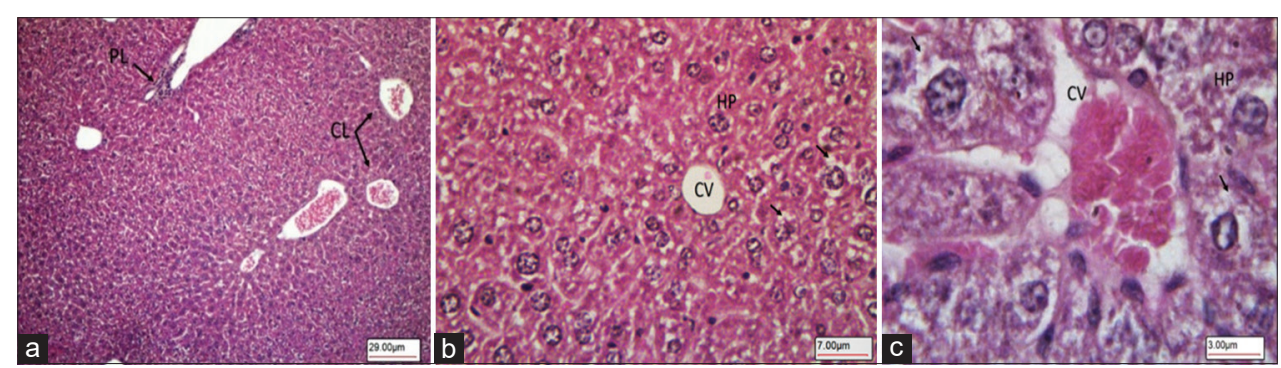

Figure-5: Diabetic control mice Group-2. (a) Liver tissue architecture. (b) Hepatocellular damage in the form of increased vacuolation in the cytoplasm of hepatocytes appeared as indistinct clear vacuoles (arrows) indicating possible glycogen infiltration and accumulation in diabetes. CV, HP. (c) Higher magnification image showing vacuoles in the cytoplasm of hepatocytes (arrows) $(100 \times, 400 \times$, and $1000 \times$, respectively). CV $=$ Central vein, HP=Hepatocytes.

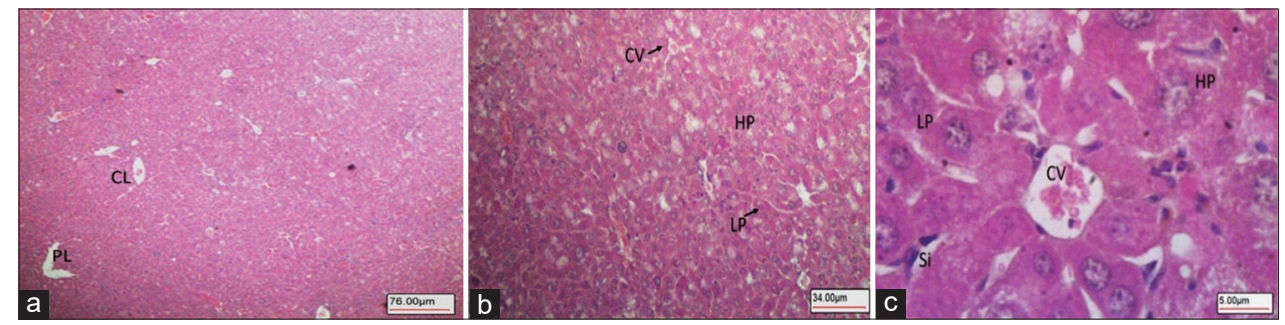

Figure-6: Diabetic mice IP treated with KBA Group-3. (a) Liver tissue appeared with normal architecture of CL and PL. (b) Normal HPs arranged in normal plates (LP) around CV. (c) High magnification showing normal HPs with mild vacuolation (HP) arranged in normal plates (LPS) around CV) Liver Si $(40 \times, 400 \times$, and $600 \times$, respectively). IP=Intraperitoneal, $\mathrm{KBA}=11$-Keto- $\beta$-boswellic acid, $\mathrm{CL}=$ Classical lobules, $\mathrm{PL}=$ Portal lobules, HPs=Hepatocytes, $\mathrm{LP}=$ Liver hepatocyte plates, $\mathrm{CV}=$ Central vein, $\mathrm{Si}=$ Sinusoids.

accumulation within the enterocytes, and saturable kinetics [31].

In this study, a rapid and inexpensive detection technique using FTIR reflectance spectroscopy coupled with a multivariate PCA method was developed to evaluate the solubility of KBA obtained from the gum resin of Omani frankincense in the liver of STZinduced diabetic mice. This novel method demonstrated that the combination of FTIR reflectance spectroscopy and PCA was a reliable and accurate technique for the visualization of similarities and differences among the three groups' mice liver tissue samples based on the solubility of KBA; it also showed a clear effect of KBA on treatments. The concentrations of KBA demonstrated distinct elevated peaks in the consolidated FTIR reflectance spectroscopy and PCA spectrum in the treated group. This result indicated that the concentration of KBA improved after IP treatment, which proved to be an effective route of administration, leading to increased KBA bioavailability. Several studies have used different approaches to enhance BA bioavailability [32,33], and many have reported the biologic effects of drugs after IP administration in animal models, suggesting the bioavailability of these large molecules as administered by said route $[34,35]$. Compared with the oral route of administration, the IP route has been proven to be more effective because it allows for a faster rate and a wider extent of drug absorption followed by the intramuscular route [36]. Pharmacological agents administered intraperitoneally are exposed to a large surface area, which leads to their rapid and efficient absorption [37].
The results of this study revealed that the high solubility of KBA in the animal model after IP treatment led to significant recovery of liver tissues and demonstrated the effect of KBA on histological alterations in the liver of STZ-induced diabetic mice. STZ is a naturally occurring compound that selectively destroys $\beta$-cells and induces alkylation of DNA, resulting in rapid necrosis, which leads to hyperglycemia [38]. Fresh liver tissue samples that were used for the solubility test were also used for histological examination. The histological findings for the diabetic mice showed classical and portal lobules in the liver tissues with histopathological changes, including hepatocellular damage in the form of increased vacuolation in the cytoplasm of hepatocytes appearing as indistinct clear vacuoles, which indicated glycogen infiltration and accumulation; these results are similar to previously reported findings [39]. After IP treatment with KBA, the liver tissues of diabetic mice markedly improved, with normal structures of hepatocytes exhibiting mild vacuolation in their cytoplasm. These results suggest that the active derivative of KBA from the gum resin of frankincense has a protective effect on the morphology of the liver of diabetic mice by significantly reducing hypoglycemia caused by STZ. Several studies have confirmed the effectiveness of KBA for diabetes and in suppressing the development of the disease in STZ-induced diabetic mice by triggering an immune response mediated by cytokines $[12,19,40]$. Furthermore, BAs have been reported to minimize liver complications and to provide a degree of protection against them [41]. Histological findings on liver tissues revealed that KBA has a protective effect 
against diabetes-induced damage [42]. The novel method herein described using FTIR reflectance spectroscopy coupled with PCA facilitated high segregation among the three groups' mice liver tissues based on the solubility of KBA, which significantly improved after IP treatment, leading to significant recovery of the liver and hepatocytes.

\section{Conclusion}

This study showed that FTIR reflectance spectroscopy coupled with PCA could be deployed as a rapid, inexpensive, and non-destructive detection method to examine the solubility of KBA in the liver of diabetic mice. BAs have been reported to effectively treat several inflammatory diseases, including diabetes. However, these active derivatives have low absorption in humans and rodents due to their poor water solubility. This limitation should be factored in when planning strategies to improve KBA solubility and its effectiveness in the treatment of DM. This study also showed that KBA has a curative potential as an antidiabetic and hepatic protective agent against STZ-induced diabetes damage by contributing to the regeneration of hepatocytes, thus providing supporting evidence for the high solubility of KBA in the animal model after IP treatment and the resulting significant recovery of liver tissues.

\section{Authors' Contributions}

IA, FM, GA, and SA: Conceived the experimental design. IA, GA, FM, SK, JA, and JS: Performed the experiment, data observation, and acquisition. IA, FM, ITK, AA, AbA, AH, SK, and BA: Analyzed and interpreted the data. IA, FM, AH, and ITK: Wrote the paper. All authors reviewed the manuscript, read, and approved the final manuscript.

\section{Acknowledgments}

The authors are thankful to Laboratory for Stem Cells and Regenerative Medicine at Natural and Medical Sciences Research Centre and DARIS Research Centre, University, University of Nizwa, Oman, for providing the necessary facilities for this study. This study was funded by University of Nizwa, Sultanate of Oman (Grant no. 26/DRC/GP/UON/2017-18).

\section{Competing Interests} interests.

The authors declare that they have no competing

\section{Publisher's Note}

Veterinary World remains neutral with regard to jurisdictional claims in published institutional affiliation.

\section{References}

1. Baynes, H.W. (2015) Classification, pathophysiology, diagnosis and management of diabetes mellitus. J. Diabetes Metab., 6(5): 1-9.

2. International Diabetic Federation. (2020) Available from: https://www.idf.org/our-network/regions-members/middleeast-and-north-africa/members/42-oman.html. Retrieved on 02-06-2021.

3. Middha, S.K., Usha, T. and Pande, V. (2014) Letter to the editor: Pomegranate peel attenuates hyperglycemic effects of alloxan-induced diabetic rats. EXCLI J., 13: 223-224.

4. Shaikh, H. and Shrivastava, V.K. (2014) Effects of streptozotocin induced diabetes mellitus Type 1 on the rat brain antioxidant status and activity of acetylcholinesterase: A novel and potential treatment by Vitex negundo. Int. J. Pharm., 6(10): 252-256.

5. Al-Haddad, R., Karnib, N., Assaad, R.A., Bilen, Y., Emmanuel, N., Ghanem, A., Younes, J., Zibara, V., Stephan, J.S. and Sleiman, S.F. (2016) Epigenetic changes in diabetes. Neurosci. Lett., 625: 64-69.

6. Cotoi, C.G. and Quaglia, A. (2016) Normal liver anatomy and introduction to liver histology. In: Textbook of Pediatric Gastroenterology, Hepatology and Nutrition. Springer, Cham. p609-612.

7. Mohamed, J., Nafizah, A.N., Zariyantey, A.H. and Budin, S.B. (2016) Mechanisms of diabetes-induced liver damage: The role of oxidative stress and inflammation. SQU Med. J., 16(2): e132.

8. Targher, G., Lonardo, A. and Byrne, C.D. (2018) Nonalcoholic fatty liver disease and chronic vascular complications of diabetes mellitus. Nat. Rev. Endocrinol., 14(2): 99-114.

9. Al-Yasiry, A.R. and Kiczorowska, B. (2016) Frankincense-therapeutic properties. Postepy Hig. Med. Dosw., 70: 380-391.

10. Sabra, S.M. and Al-Masoudi, L.M. (2014) The effect of using frankincense (Boswellia sacra) chewing gum on the microbial contents of buccal/oral cavity, Taif, KSA. J. Dent. Med. Sci., 13(4): 77-82.

11. Ni, X., Suhail, M.M., Yang, Q., Cao, A., Fung, K.M., Postier, R.G., Woolley, C., Young, G., Zhang, J. and Lin, H.K. (2012) Frankincense essential oil prepared from hydrodistillation of Boswellia sacra gum resins induces human pancreatic cancer cell death in cultures and in a xenograft murine model. BMC Complement. Altern. Med., 12: 253.

12. Ammon, H.P. (2019) Boswellic extracts and 11-keto-ß-boswellic acids prevent Type 1 and Type 2 diabetes mellitus by suppressing the expression of proinflammatory cytokines. Phytomedicine, 63: 153002.

13. Al-Harrasi, A., Ali, L., Ceniviva, E., Al-Rawahi, A., Hussain, J., Hussain, H., Rehman, N.U., Abbas, G. and Al-Harrasi, R. (2013) Antiglycation and antioxidant activities and HPTLC analysis of Boswellia sacra Oleogum resin: The sacred frankincense. Trop. J. Pharma. Res., 12(4): 597-602.

14. Al-Harrasi, A., Ali, L., Hussain, J., Rehman, N.U., Ahmed, M. and Al-Rawahi, A. (2014) Analgesic effects of crude extracts and fractions of Omani frankincense obtained from traditional medicinal plant Boswellia sacra on animal models. Asian Pac. J. Trop. Med., 7: S485-S490.

15. Saraswati, S. and Agrawal, S.S. (2012) Antiangiogenic and cytotoxic activity of boswellic acid on breast cancer MCF-7 cells. Biomed. Prev. Nutr., 2(1): 31-37.

16. Ahmed, H.H., Ahmed, A., Abd-Rabou, A.Z., Hassan, S. and Kotob, E. (2015) Phytochemical analysis and anticancer investigation of Boswellia serrata bioactive constituents in vitro. Asian Pac. J. Cancer Prev., 16(16): 7179-7188.

17. Pengzong, Z., Yuanmin, L., Xiaoming, X., Shang, D., Wei, X., Zhigang, L., Dongzhou, D., Wenjing, Y., Jianbiao, Y., Yang, X. and Xia, L. (2019) Wound healing potential of the standardized extract of Boswellia serrata on experimental diabetic foot ulcer via inhibition of inflammatory, angiogenetic and apoptotic markers. Planta Med., 85(8): 657-669.

18. Shehata, A., Quintanilla-Fend, L., Bettio, S., Jauch, J., Scior, T., Scherbaum, W. and Ammon, H. (2015) 
11 -keto- $\beta$-boswellic acids prevent development of autoimmune reactions, insulitis and reduce hyperglycemia during induction of multiple low-dose streptozotocin (MLD-STZ) diabetes in mice. Horm. Metab. Res., 47(6): 463-469.

19. Wang, H., Zhang, C., Wu, Y., Ai, Y., Lee, D.Y. and Dai, R. (2014) Comparative pharmacokinetic study of two boswellic acids in normal and arthritic rat plasma after oral administration of Boswellia serrate extract or Huo Luo Xiao Ling Dan by LC-MS. Biomed. Chrom., 28(10): 1402-1408.

20. Hüsch, J., Bohnet, J., Fricker, G., Skarke, C., Artaria, C., Appendino, G., Schubert-Zsilavecz M. and AbdelTawab, M. (2013) Enhanced absorption of boswellic acids by a lecithin delivery form (phytosome ${ }^{\circledR}$ ) of Boswellia extract. Fitoterapia, 84: 89-98.

21. Büchele, B. and Simmet, T. (2003) Analysis of 12 different pentacyclic triterpenic acids from frankincense in human plasma by high performance liquid chromatography and photodiode array detection. J. Chromatogr. B Analyt. Technol. Biomed. Life Sci., 795(2): 355-362.

22. Sharma, S., Thawani, V., Hingorani, M., Shrivastava, V., Bhate, R. and Khiyani, R. (2004) Pharmacokinetic study of 11 Keto $\beta$-boswellic acid. Phytomedicine, 11(2): 255-260.

23. Berthomieu, C. and Hienerwadel, R. (2009) Fourier transform infrared (FTIR) spectroscopy. Photosynth. Res., 101(2-3): 157-170.

24. Ruah, M.E., Rasaruddin, N.F., Fong, S.S. and Jaafar, M.Z. (2014) Data preprocessing methods of FT-NIR spectral data for the classification cooking oil. Am. Inst. Phys. Conf. Proc., 1635: 890-897.

25. Ginter, E. and Simko, V. (2012) Type 2 diabetes mellitus, pandemic in $21^{\text {st }}$ century. Adv. Exp. Med. Biol., 771(3): 42-50.

26. Ammon, H.P.T. (2016) Boswellic acids and their role in chronic inflammatory diseases. Adv. Exp. Med. Biol., 928: 291-327.

27. Karlina, M.V., Pozharitskaya, O.N., Kosman, V.M. and Ivanova, S.A. (2007) Bioavailability of boswellic acids: In vitro/in vivo correlation. Pharm. Chem. J., 41(11): 569-572.

28. Kröger, P., Rambod, D., Gunter, P., Eckert, J.K., Dietrich, A., Volmer, U.B., Walter, E., Muller, M.K., SchubertZsilavecz, M. and Abdel-Tawab, M. (2008) Metabolism of boswellic acids in vitro and in vivo. Drug Metab. Dispos., 36(6): 1135-1142.

29. Bagul, P., Khomane, K.S. and Bansal, A.K. (2014) Investigating permeability related hurdles in oral delivery of 11-keto-beta-boswellic acid. Int. J. Pharm., 464(1-2): 104-110.

30. Skarke, C., Kuczka, K., Tausch, L., Werz, O., Rossmanith, T., Barrett, J.S., Harder, S., Holtmeier, W. and Schwarz, J.A. (2012) Increased bioavailability of 11-keto-beta-boswellic acid following single oral dose frankincense extract administration after a standardized meal in healthy male volunteers: Modeling and simulation considerations for evaluating drug exposures. J. Clin. Pharm., 52(10): 1592-1600.

31. Du, Z., Liu, Z., Ning, Z., Liu, Y., Song, Z., Wang, C. and
Lu, A. (2015) Prospects of boswellic acids as potential pharmaceutics. Planta Med., 81(4): 259-271.

32. Lee, B., Clarke, D., Al-Ahmad, A., Kahle, M., Parham, C., Auckland, L., Shaw, C., Fidanboylu, N., Orr, W.A., Ogunshola, O., Fertala, A., Thomas, S.A. and Gregory, J.B. (2001) Perlecan domain V is neuroprotective and proangiogenic following ischemic stroke in rodents. J. Clin. Invest., 121(8): 3005-3023.

33. Zhang, B., Dai, J., Wang, H., Wei, H., Zhao, J., Guo, Y. and Fan, K. (2014) Antiosteopontin monoclonal antibody prevents ovariectomy-induced osteoporosis in mice by promotion of osteoclast apoptosis. Biochem. Biophysical. Res. Commun., 452(3): 795-800.

34. Wangler, N.J., Jayaraman, S., Zhu, R., Mechref, Y., Abbruscato, T.J., Bickel, U. and Karamyan, V. (2016) Preparation and preliminary characterization of recombinant neurolysin for in vivo studies. J. Biotechnol., 234: 105-115.

35. Chang, R., Al-Maghribi, A., Vanderpoel, V., Vasilevko, V., Cribbs, D.H., Boado, R., Pardridge, W.M. and Sumbria, R.K. (2018) Brain penetrating bifunctional erythropoietin-transferrin receptor antibody fusion protein for Alzheimer's disease. Mol. Pharm., 15(11): 4963-4973.

36. Wolfensohn, S.E. and Lloyd, M.H. (1994) Aleutian disease in laboratory ferrets. Vet. Record, 134(4): 100.

37. Al Shoyaib, A., Sabrina, R.A. and Vardan, T.K. (2020) Intraperitoneal route of drug administration: Should it be used in experimental animal studies? Pharm. Res., 37(1): 12.

38. Chinedum, O.E., Eleazu, K., Chukwuma, S. and Essien, U.N. (2013) Review of the mechanism of cell death resulting from streptozotocin challenge in experimental animals, its practical use and potential risk to humans. $J$. Debates. Metab. Disord., 12(1): 60.

39. Saad, Entsar A, Mohamed M. Hassanien, Maha A. El-Hagrasy, Kholoud H. Radwan. (2015) Antidiabetic, hypolipidemic and antioxidant activities and protective effects of Punica granatum peels powder against pancreatic and hepatic tissues injuries in streptozotocin induced IDDM in rats. Int. J. Pharm. Pharm. Sci., 7(7): 397-402.

40. Ammon, H., Shehata, A.M. and Quintanilla-Fend, L. (2011) 11-keto-beta-boswellic acid (KBA) suppresses development of insulitis-apoptosis of pancreatic islets and elevation of proinflammatory interleukins in multiple low dose streptozotocin (MLD-STZ)-induced diabetes of mice. Am. Diabetes Assoc., 60: A278-A278.

41. Iram, F., Khan, S.A. and Husain, A. (2017) Phytochemistry and potential therapeutic actions of boswellic acids: A mini-review. Asian Pac. J. Trop. Biomed., 7(6): 513-523.

42. Azemi, M.E., Namjoyan, F., Khodayar, M.J., Ahmadpour, F., Padok, A.D. and Panahim, M. (2012) The antioxidant capacity and antidiabetic effect of Boswellia serrata triana and planch aqueous extract in fertile female diabetic rats and the possible effects on reproduction and histological changes in the liver and kidneys. J. Nat. Pharm. Prod., 7(4): 168-175. 